\title{
Aplicación de la escala MNA en adultos mayores de la parroquia Quingeo - Cuenca, 2018
}

\section{Application of the MNA scale in older adults in the parish of Quingeo - Cuenca, 2018}

\author{
Gabriela Liliana Jarro Calle*1, Fanny Cecilia Rodríguez Quezada ${ }^{1}$, Edison Gustavo Moyano Brito ${ }^{1}$, Rommel \\ Patricio Guachizaca Uyaguari ${ }^{1}$ \\ ${ }^{1}$ Universidad Católica de Cuenca, Ecuador \\ *gljarroc55@est.ucacue.edu.ec
}

DOI: https://doi.org/10.26871/killkanasalud.v4i4.746

\begin{abstract}
Resumen
Los problemas relacionados con la nutrición de las personas mayores han cobrado una especial relevancia en la actualidad y han sido un tema de estudio para los profesionales de la salud; la OMS ha señalado a este grupo de la población como nutricionalmente vulnerable debido al déficit fisiologico alcanzado. En el año 2014; cifras estimadas mundialmente; revelan que había aproximadamente 462 millones de adultos con insuficiencia ponderal y 1900 millones de personas con sobrepeso u obesidad. Objetivo: Valorar el estado nutricional del adulto mayor mediante la escala de medición mínima nutricional "MNA" aplicada a los integrantes del Club de Adultos Mayores de la Parroquia Quingeo, cantón Cuenca en el periodo de enero a septiembre del 2018. Métodos y Materiales: La siguiente investigación fue cuantitativa, de nivel descriptivo de corte transversal, se trabajó con la totalidad de la población, 130 adultos mayores. La recolección de los datos se realizó con la Escala de Evaluación Mínima Nutricional, consta de 18 ítems de opción múltiple, se integró datos sociodemográficos y antropométricos. Resultados: El MNA muestra que el $60 \%$ se encuentra en riesgo de malnutrición, el $40 \%$ está desnutrido. El IMC, el 46,2\% normo peso, $40 \%$ con clasificación de bajo peso y con el $11.5 \%$ sobrepeso, por último, obesidad con un porcentaje mínimo de 2,3\%. Conclusiones: El estado nutricional muestra que un alto porcentaje de la población está en riesgo de desnutrición según el MNA, menos de la mitad tienen bajo peso y un porcentaje similar está en normo peso con valores de IMC.
\end{abstract}

Palabras clave: adulto mayor, estado nutricional, malnutrición, obesidad, sobrepeso, desnutrición.

\begin{abstract}
The problems related to the nutrition of the elderly have become especially relevant today and have been a subject of study for health professionals; The WHO has identified this group of the population as nutritionally vulnerable due to the physiological deficit achieved. In 2014; estimated figures worldwide; reveal that there were approximately 462 million underweight adults and 1.9 billion people who were overweight or obese. Objective: To assess the nutritional status of the elderly through the minimum nutritional measurement scale "MNA" applied to the members of the Club of Older Adults of Quingeo Parish, Cuenca canton in the period from January to September 2018. Methods and Materials: The The following research was quantitative, descriptive level, cross-sectional, and worked with the entire population, 130 older adults. The data collection was carried out with the Minimum Nutritional Assessment Scale, it consists of 18 multiplechoice items, sociodemographic and anthropometric data were integrated. Results: The MNA shows that 60\% are at risk of malnutrition, $40 \%$ are malnourished. BMI, 46.2\% normal weight, $40 \%$ classified as underweight and with $11.5 \%$ overweight, and finally, obesity with a minimum percentage of 2.3\%. Conclusions: The nutritional status shows that a high percentage of the population is at risk of malnutrition according to the MNA, less than half are underweight and a similar percentage is in normal weight with BMI values.
\end{abstract}

Keywords: elderly, nutritional status, malnutrition, obesity, overweight, malnutrition.

\section{Introducción}

La Organización Mundial de la Salud se refiere al envejecimiento como el resultado de la acumulación de daños y deterioro a nivel molecular, celular a largo de la vida dando como consecuencia el declive biológico y de las capacida- des mentales y físicas, aumentando el riesgo de enfermedad y finalmente la muerte. ${ }^{1-4}$ En los adultos mayores el declive del estado nutricional perjudica significativamente a la calidad de vida, aumenta la morbilidad sobre todo por aumento de enfermedades agudas y crónicas. ${ }^{5,6}$ 
Demográficamente el desarrollo de envejecimiento es el efecto de los cambios en las tasas de fecundidad, mortalidad, migración y la relación entre estas. El Ecuador vive un proceso de transición demográfica, caracterizado por el envejecimiento poblacional, este fenómeno de envejecimiento demográfico que se acelerara aún más en las próximas décadas. $^{7}$

El estado de nutrición de los adultos mayores está determinado por el consumo y la demanda de nutrientes, este se ve afectado por estilo de vida, el entorno en donde se desenvuelve el individuo y su situación socioeconómica. ${ }^{8}$

El propósito de la presente investigación es valorar el estado nutricional de los adultos mayores, de esta manera, clasificando el estado nutricional en el que se encontraban los participantes del club de Adultos mayores, identificando factores de riesgo, interviniendo de forma promocional sobre un apropiado consumo de nutrientes, mejorando el estado nutricional y con ello disminuir la morbilidad a causa de una mala nutrición.

Con los resultados obtenidos se pretende influir en la búsqueda de estrategias para mejorar en nivel nutricional de esta población vulnerable, para que el personal de salud, familia y actores políticos tomen un papel activo y participativo el empoderamiento comunitario, para encaminar las diferentes alternativas para resolver problemas y necesidades que afectan a los adultos mayores.

\section{Materiales y métodos}

Se aplico un enfoque cuantitativo de nivel descriptivo no experimental con temporalidad prospectiva y de corte transversal. El universo de estudio estuvo constituido por 130 adultos mayores entre hombres y mujeres que acuden al Club del Adulto Mayor del Centro de Salud Quingeo de la Parroquia Quingeo, área rural del cantón Cuenca provincia del Azuay, de enero a septiembre del año 2018. Se excluyo a las personas que no deseaban participar, a las que no firmaron el consentimiento informado, a las que no cumplían los 65 años y a las que, a pesar de firmar el consentimiento, expresaron su deseo de retirarse del estudio.

No se requirió el cálculo de una muestra, puesto que se trabajó con todos los individuos que conforman el universo, considerando los criterios de inclusión y exclusión.

Luego de socializar el proyecto de investigación se aplicó el método de una encuesta con parámetros dietéticos incluyendo datos demográficos; después se procedió a recolectar las medidas antropométricas, para lo cual se les pidió a los participantes quedarse vestidos únicamente con prendas ligeras (camisa y pantalón, blusa y falda o pantalón); y se usaron un tallímetro graduado en centímetros con una precisión de 0,5 cm (SECA 206 Hamburgo, Alemania); báscula mecánica ASTRA precisión de 100g y cinta métrica graduada en centímetros no elástica con una precisión de 1mm (SECA 206 Hamburgo, Alemania).

En cuanto al peso, se les dio algunas indicaciones como: portar prendas ligeras, despojarse de objetos como celular, carteras, llaves o monedas, colocarse de pie en la báscula con los talones juntos formando un ángulo de $45^{\circ}$, haber ido al baño previamente y se indago en cada uno si tomó o no el desayuno para realizar la respectiva resta del peso siendo este de 400 a 600 gr.

Para la talla se les indicó colocarse frente al tallímetro con la cabeza, hombros y cadera pegados a la pared, talones juntos formando un ángulo de $45^{\circ}$, los brazos colgando naturalmente y mantenerse firmes con la vista al frente.

El perímetro braquial; se registró dejando libre de prendas el brazo, en posición sentado o de pie con los brazos colgando naturalmente. El perímetro de pantorrilla; se indicó estar de pie con las piernas separadas 20 centímetros y dejar libre de prendas de vestir el espacio desde la rodilla hasta el pie.

Para la recolección de los datos informáticos se usó como instrumento el formulario nutricional Evaluación Mínima Nutricional o MNA (Mini Nutricional Assessment). Este formulario consta de 18 preguntas divididas en 4 partes: Primero, medidas antropométricas como índice de masa corporal, circunferencia branquial, de pantorrilla y pérdida de peso. Segundo, evaluación global del estilo de vida, polimedicación y movilidad. Tercero, ingesta de líquidos, alimentación y autonomía para alimentarse. Cuarto, evaluación subjetiva es decir autoevaluación nutricional.

Con estos datos se procede a ubicar a los participantes en 3 grupos: 1.) Desnutrición (inferior a 17 puntos). 2.) Riesgo de malnutrición (entre 17 a 23,5 puntos). 3.) Normo peso (24 a 30 puntos).

El procesamiento de datos se realizó con el programa Statistical Package For the Social Sciences "SPSS" versión 24.0, donde se realizó el análisis estadístico. Las medidas que se emplearon fueron el porcentaje, la media y el promedio.

\section{Resultados}

La población de estudio estuvo conformada por 130 adultos mayores siendo $85(65,4 \%)$ de sexo femenino y 45 $(34.6 \%)$ de sexo masculino. El grupo etario que predomino fue el de 70 a 79 años con el $58.46 \%(n=76)$, seguido por 65 a 69 años, con el $37.69 \%(n=49)$, luego el de 80 a 84 años $2.32 \%(n=3)$ y por último mayores de 85 años el $1,53 \%$ $(\mathrm{n}=2)$. (tabla 1)

Los datos para el cálculo del índice de masa corporal demuestran que el $46.2 \%(n=60)$ se ubican con normo peso, seguido del $40 \%(\mathrm{n}=52)$ con bajo peso, el $11.5 \%$ $(n=15)$ con sobrepeso y el $2.3 \%(n=3)$ con obesidad. (tabla 2)

Por otra parte, el $64.6 \%(n=84)$ no sabe si ha perdido peso en los últimos 3 meses, el $32.3 \%(n=42)$ manifiesta pérdida de peso entre 1 a $3 \mathrm{~kg}$ de peso y el $3.1 \%(\mathrm{n}=4)$ ha perdido más de $3 \mathrm{~kg}$ de peso. (tabla 3 )

Se evidencio que existe un alto porcentaje de individuos con anorexia moderada $80 \%(n=104)$; y tan solo el $1.5 \%$ $(n=2)$ con anorexia grave; el resto de la población $18.5 \%$ $(n=24)$ no presenta anorexia. (tabla 4) 
Los adultos mayores que ingieren 2 comidas completas por día corresponden al 76,2\% $(n=99)$ y el $23,8 \%(n=31)$ una comida completa por día. (tabla 5)

El 93.1\% ( $\mathrm{n}=121)$ de los individuos consume 2 alimentos con base proteica por día o semana, seguido por el $6.2 \%$ $(\mathrm{n}=8)$ que consumen 1 producto. y el $0,8 \%(\mathrm{n}=1)$ consume 3 productos. (tabla 6 )

De igual manera se evaluó el consumo de frutas y verduras denotando que el $70.8 \%(n=92)$ no consume vitaminas y solo el $9.2 \%$ ? si lo hace, estos resultados están relacionados con el nivel socioeconómico del participante. (tabla 7).

La autopercepción nutricional de los participantes demostró que el $93.1 \%(n=121)$ no sabe cómo se encuentra nutrido o considera que tiene malnutrición moderada; el $4.6 \%(n=6)$ no tiene problemas de nutrición y el $2.3 \%$ $(n=3)$ tiene malnutrición grave. (tabla 8)

Las enfermedades agudas o estrés psicológico estuvieron presentes en el $96.2 \%(n=125)$ de los individuos. La demencia o depresión moderada se repite en los participantes con malnutrición y en más de la mitad de la población, 58 participantes con depresión moderada y en menor frecuencia con 5 participantes con depresión grave.

La interpretación según el MNA muestra que más de la mitad de la población $60,0 \%(n=78)$ se encuentra en riesgo de desnutrición y el 40,0\% ( $n=52)$ en desnutrición. (tabla 9)

Tabla 1: Distribución de los participantes según la edad

\begin{tabular}{lcc}
\hline Edad & Frecuencia & Porcentaje \% \\
\hline 65 a 69 & 49 & 37,69 \\
70 a 79 & 76 & 58,46 \\
80 a 84 & 3 & 2,32 \\
Más de 85 & 2 & 1,53 \\
Total & 130 & 100,0 \\
\hline
\end{tabular}

Tabla 2: Distribución de los participantes según el índice de masa corporal

\begin{tabular}{lcc}
\hline IMC & Frecuencia & Porcentaje \% \\
\hline IMC menor a 22 Bajo peso & 52 & 40,0 \\
IMC de 22 a 27 Eutrófico Normo peso & 60 & 46,2 \\
IMC de 27 a 32 Sobrepeso & 15 & 11,5 \\
IMC mayor a 32 Obesidad & 3 & 2,3 \\
Total & 130 & 100,0 \\
\hline
\end{tabular}

Tabla 3: Distribución de los participantes según perdida reciente de peso

\begin{tabular}{lcc}
\hline Perdida reciente de peso $(<$ tres meses) & Frecuencia & Porcentaje \% \\
\hline Perdida de peso $>3 \mathrm{Kg}$ & 4 & 3,1 \\
No lo sabe & 84 & 64,6 \\
Pérdida de peso entre 1 y $3 \mathrm{Kg}$ & 42 & 32,3 \\
Total & 130 & 100,0 \\
\hline
\end{tabular}

Tabla 4: Distribución de los participantes según anorexia

\begin{tabular}{lcc}
\hline $\begin{array}{l}\text { ¿Ha perdido el apetito? ¿Ha comido } \\
\text { menos por falta de apetito, problemas } \\
\text { digestivos, dificultades de masticación o }\end{array}$ & Frecuencia & Porcentaje \% \\
$\begin{array}{l}\text { deglución en los últimos 3 meses? } \\
\text { Anorexia grave }\end{array}$ & \\
Anorexia moderada & 104 & 1,5 \\
Sin anorexia & 24 & 80,0 \\
Total & 130 & 18,5 \\
\hline
\end{tabular}

Tabla 5: Distribución de los participantes según el número de comidas diarias

\begin{tabular}{lcc}
\hline $\begin{array}{l}\text { ¿Cuántas comidas completas toma al } \\
\text { día? }\end{array}$ & Frecuencia & Porcentaje \% \\
\hline 1 comida & 31 & 23,8 \\
2 comidas & 99 & 76,2 \\
Total & 130 & 100,0 \\
\hline
\end{tabular}

Tabla 6: Distribución de los participantes según el consumo de alimentos con contenido proteico

¿Consume el paciente (si o no)?

- ¿Productos lácteos al menos una vez al día?

- ¿Huevos o legumbres 1 o 2 veces a la Frecuencia Porcentaje \% semana?

- ¿Carne, pescado o aves diariamente?

\begin{tabular}{lcc}
\hline 0 o 1 síes & 8 & 6,2 \\
2 síes & 121 & 93,1 \\
3 síes & 1 & 0,8 \\
Total & 130 & 100,0 \\
\hline
\end{tabular}

Tabla 7: Distribución de los participantes según el consumo de frutas y verduras

\begin{tabular}{lcc}
\hline ¿Consume frutas y verduras al menos & Frecuencia & Porcentaje \% \\
dos veces al día? & 92 & 70,8 \\
\hline No & 38 & 29,2 \\
Si & 130 & 100,0 \\
Total & \\
\hline
\end{tabular}

Tabla 8: Distribución de los participantes según la autopercepción nutricional

\begin{tabular}{lcc}
\hline ¿Considera el paciente que está bien nu- & Frecuencia & Porcentaje \% \\
trido? & 3 & 2,3 \\
\hline Malnutrición grave & 121 & 93,1 \\
No lo sabe o malnutrición moderada & 6 & 4,6 \\
Sin problemas de nutrición & 130 & 100,0 \\
Total & & \\
\hline
\end{tabular}

Tabla 9: Distribución de los participantes según la clasificación MNA

\begin{tabular}{lcc}
\hline $\begin{array}{l}\text { ¿Considera el paciente que está bien nu- } \\
\text { trido? }\end{array}$ & Frecuencia & Porcentaje \% \\
\hline Menor a 17 desnutrición & 52 & 40,0 \\
De 17 a 23 riesgo de desnutrición & 78 & 60,0 \\
Total & 130 & 100,0 \\
\hline
\end{tabular}


Tabla 10: Comparación entre MNA e IMC

\begin{tabular}{|c|c|c|c|c|c|c|c|}
\hline & \multicolumn{4}{|c|}{ Clasificación según IMC } & \multirow{2}{*}{ Total } \\
\hline & & & $\begin{array}{l}\text { IMC menor a } \\
22 \text { Bajo peso }\end{array}$ & $\begin{array}{l}\text { IMC de } 22 \text { a } \\
27 \text { Eutrófico }\end{array}$ & $\begin{array}{l}\text { IMC de } 27 \text { a } \\
32 \text { Sobrepeso }\end{array}$ & $\begin{array}{l}\text { IMC mayor a } \\
32 \text { Obesidad }\end{array}$ & \\
\hline \multirow{8}{*}{$\begin{array}{l}\text { Clasificación } \\
\text { según la } \\
\text { Puntuación } \\
\text { Global }\end{array}$} & \multirow{4}{*}{$\begin{array}{l}\text { Menor a } 17 \\
\text { desnutrición }\end{array}$} & Recuento & 35 & 13 & 3 & 0 & 51 \\
\hline & & $\begin{array}{l}\text { \% dentro de Clasificación según la Pun- } \\
\text { tuación Global }\end{array}$ & $68,6 \%$ & $25,5 \%$ & $5,9 \%$ & $0,0 \%$ & $100,0 \%$ \\
\hline & & $\%$ dentro de Clasificación según IMC & $68,6 \%$ & $21,7 \%$ & $20,0 \%$ & $0,0 \%$ & $39,5 \%$ \\
\hline & & $\%$ del total & $27,1 \%$ & $10,1 \%$ & $2,3 \%$ & $0,0 \%$ & $39,5 \%$ \\
\hline & \multirow{4}{*}{$\begin{array}{l}\text { De } 17 \text { a } 23 \\
\text { riesgo de } \\
\text { desnutrición }\end{array}$} & Recuento & 16 & 47 & 12 & 3 & 78 \\
\hline & & $\begin{array}{l}\text { \% dentro de Clasificación según la Pun- } \\
\text { tuación Global }\end{array}$ & $20,5 \%$ & $60,3 \%$ & $15,4 \%$ & $3,8 \%$ & $100,0 \%$ \\
\hline & & $\%$ dentro de Clasificación según IMC & $31,4 \%$ & $78,3 \%$ & $80,0 \%$ & $100,0 \%$ & $60,5 \%$ \\
\hline & & $\%$ del total & $12,4 \%$ & $36,4 \%$ & $9,3 \%$ & $2,3 \%$ & $60,5 \%$ \\
\hline \multirow{4}{*}{\multicolumn{2}{|c|}{ Total }} & Recuento & 51 & 60 & 15 & 3 & 129 \\
\hline & & $\begin{array}{l}\% \text { dentro de Clasificación según la Pun- } \\
\text { tuación Global }\end{array}$ & $39,5 \%$ & $46,5 \%$ & $11,6 \%$ & $2,3 \%$ & $100,0 \%$ \\
\hline & & $\%$ dentro de Clasificación según IMC & $100,0 \%$ & $100,0 \%$ & $100,0 \%$ & $100,0 \%$ & $100,0 \%$ \\
\hline & & $\%$ del total & $39,5 \%$ & $46,5 \%$ & $11,6 \%$ & $2,3 \%$ & $100,0 \%$ \\
\hline
\end{tabular}

\section{Discusión}

El crecimiento poblacional de adultos mayores, así como la esperanza de vida es mayor para el género femenino 9,10

El nivel de escolaridad de los participantes se asoció con el estado nutricional de la población, la baja instrucción educativa está relacionada con la desnutrición en un $95 \%{ }^{11}$

En cuanto a la clasificación nutricional, existe una mayor prevalencia porcentual del riesgo nutricional, seguido por la malnutrición y finalmente la desnutrición, estos resultados concuerdan con los estudios de: Calderón et al. 2104; Deossa et al. 2016; Choque 2013 y Monsalve et al. 2011. Es necesaria la intervención del personal de salud en este contexto, vale la pena mencionar que padecer desnutrición o riesgo de desnutrición repercute en la salud y la calidad de vida de los adultos mayores ${ }^{9,12,13}$

La clasificación según el índice de masa corporal IMC, ubica en mayor porcentaje a normo peso (46.2\%), seguido de bajo peso $(40 \%)$, sobrepeso $(11.5 \%)$ y finalmente obesidad (2.3\%); datos similares al estudio de González donde se puede evidenciar la misma escala de porcentajes además de niveles de desnutrición leve, desnutrición moderada y finalmente desnutrición severa. ${ }^{14,15}$

En cuanto a parámetros dietéticos se pueden relacionar una mayor prevalencia de riesgo de malnutrición con una ingesta por debajo de dos comidas completas al día $(23.8 \%)$, consumo bajo de frutas y verduras $(70.8 \%)$ así como de alimentos con base proteica por día o semana con poca frecuencia $(6.2 \%)$, datos que van en concordancia con el estudio de Deossa y González. ${ }^{12,16}$ Los alimentos de fuente de proteína y así como micronutrientes el consumo de fuente vitamínica es importante para mantener el funcionamiento del organismo y el metabolismo ya que contiene antioxidantes, vitaminas, minerales además de contener fibra son importantes para el mantenimiento de la salud y un envejecimiento saludable. ${ }^{17}$

Entre los principales hallazgos del presente estudio se encontró además que un alto porcentaje $(80.0 \%)$ refiere anorexia moderada, seguido por el grupo que desconoce si perdió peso (64.6\%), además el 96,2\% de la población ha tenido enfermedad aguda o estrés psicológico en los últimos tres meses; el $44.6 \%$ afirma tener demencia o depresión moderada y finalmente un 3,8\% tuvo depresión grave. Estos datos discrepan de un estudio realizado en España, en donde se usó el mismo instrumento para la recolección de datos en donde se reporta un bajo porcentaje de desnutrición grave $(0.4 \%)$, seguido de una malnutrición moderada $(16.1 \%)$. De igual manera no hay relación en cuanto al porcentaje de enfermedad aguda o estrés psicológico presente en solo el $27.9 \%$, depresión moderada en el $21.7 \%$ valores muy bajos en comparación al presente estudio. ${ }^{9}$

La Guía de Práctica Clínica en su apartado de cambios fisiológicos del envejecimiento menciona que la reducción del apetito se conoce como "anorexia del envejecimiento" y su prevalencia es de $21 \%$ en las personas mayores de 65 años, la conjugación de anorexia y otros factores de riesgo hacen que el adulto mayor no recupere peso y permanezca más tiempo desnutrido aumentando la morbilidad. ${ }^{18}$ Pando et al, encuentra una mayor prevalencia de depresión en mujeres adultas mayores $43 \%$ en comparación con los hombres $27.1 \% .^{19,20}$

\section{Conclusión}

Más de la mitad de la población presento riesgo de malnutrición según parámetros de MNA. El IMC evidenció que un alto porcentaje de individuos presentó normo peso, en menor frecuencia bajo peso, seguido de sobre peso y obesidad en menor frecuencia.

Al culminar la investigación se identificó que los riesgos asociados a malnutrición fueron: la presencia de anorexia moderada; la baja frecuencia de consumo de alimentos de valor proteico y vitamínico; desconocer o considerar tener malnutrición moderada; enfermedad aguda o estrés psicológico; demencia o depresión moderada y en menor frecuencia depresión grave. 


\section{Fuente de Financiamiento}

Este estudio es autofinanciado.

\section{Conflicto de Intereses}

No existen conflictos personales, profesionales, financieroso de otro tipo.

\section{Consentimiento Informado}

Los autores cuentan con el consentimiento informado de los pacientes para la investigación, la publicación del caso y sus imágenes.

\section{Referencias Bibliográficas}

1. OMS. World Health Organization. [Online].; 2015 [cited 2018 Octubre 2. Available from: https://www.afro.who. int/health-topics/ageing.

2. De Loyola. LOIOLA XXI. [Online].; 2015 [cited 2018 Octubre 2. Available from: https: 16 //loiolaxxi.wordpress.com/2015/02/12/obesidad-ysobrepeso-en-el-mundo-informe-oms/.

3. Del Avellanal Calzadilla A. Alzheimer Universal. [Online].; 2013 [cited 2018 Octubre 2. Available 17. from: https://www.alzheimeruniversal.eu/2013/10/ 01/envejecimiento-cambios-biologicos-cambiosfuncionales/.

4. Alvarado García AM, Salazar Maya AM. Análisis del concepto de envejecimiento. Gerokomos. 2014; 25(2): p. 57-62.

5. Torrejón Mendoza C, Reyna Márquez E. Estilo de vida y estado nutricional del adulto mayor. In Cress. 2012 Octubre; 3(2): p. 267-276.

6. OMS. World Health Organization. [Online].; 2014 [cited 2018 Octubre 2. Available from: https://www.who.int/ ageing/about/facts/en/.

7. Egas M. La población adulta mayor crecerá $34 \%$ a patir de 2020. El Telégrafo. 2016 Junio.

8. Rosero Otero M, Rosas Estrada GM. Valoración nutricional de las personas mayores de 60 años de la ciudad de Pasto, Colombia. Ciencia y Enfermeria. 2017 Diciembre ; 23(3).

9. Deossa Restrepo GC, Restrepo Betancur LF, Velásquez Vargas JE, Varela Álvarez D. Evaluación nutricional de adultos mayores con el Mini Nutritional Assessmenta: MNA. Universidad y Salud. 2016 Diciembre ; 18(3).

10. Social MdIEy. Ministerio de Inclusión Económica y Social. [Online]; 2017 [cited 2018 Octubre 2. Available from: https://www.inclusion.gob.ec/direccion-poblacionadulta-mayor/.

11. Tarqui Mamani C, Alvarez Dongo D, Espinoza Oriundo P, Gomez Guizado G. Estado nutricional asociado a características sociodemográficas en el adulto mayor peruano. Rev Peru Med Exp Salud Publica. 2014 Mayo; 31(3): p. 467-472.

12. Calderón Reyes ME, Ibarra Ramírez F, García J, Gómez Alonso C, Rodríguez Orozco AR. Evaluación nutricional comparada del adulto mayor en consultas de medicina familiar. Nutr Hosp. 2010; 25(4): p. 669-675.

13. Monsalve Vega MdL, Quintanilla Zamora G, Trujillo Amoroso MV. Situación nutricional de los adultos mayores que habitan en las residencias de acogida: Miguel León y Cristo Rey de la ciudad de Cuenca - 2011. Tesis pregrado. Cuenca: Unversidad de Cuenca, Facultad de Ciencias Médicas, Escuela de Tencología Médica, Área de nutrición y dietética; 2011.

4. González González JL. Evaluación del estado nutricional de un grupo de adultos mayores pertenecientes al plan nueva sonrisa dispensario Santa Francisca Romana. Tesis pregrado. Bogotá: Pontificia Universidad Javeriana, Carrera de Nutrición y Dietética; 2011.

15. De Arriba Muñoz A, López Úbeda M, Rueda Caballero C, Labarta Aizpún JI, Ferrández Longás Á. Valores de normalidad de índice de masa corporal y perímetro abdominal en población española desde el nacimiento a los 28 años de edad. Nutr Hops. 2016 Agosto; 33(4): p. 887-893.

6. De Morais C, Olìveìra B, Afonso C, Lumbers M, Raats M, De Almeida M. Nutritional risk of European elderly. European Journal of Clinical Nutrition. 2013 September; 67: p. 1215-1219.

7. Giraldo Acosta M. Evaluación del estado nutricional de $\mathrm{n}$ grupo de adultos mayores del asilo San Antonio, Honda (Tolima-Colombia). Tesis pregrado. Bogotá: Pontificia Universidad Javeriana, Carrera de Nutrición y Dietética; 2016.

8. Salud CNdETe. Evaluación y seguimiento nutricional del adulto mayor en el primer nivel de atención. Guía de referencia rápida. México D. F.: Instituto Mexicano del Seguro Social.; 2014.

19. Pando Moreno M, Aranda Beltrán C, Alfaro Alfaro N, Mendoza Roaf P. Prevalencia de depresion en adultos mayores en poblacion urbana. Revista Española de Geriatría y Gerontología. 2001; 36(3): p. 140-144.

20. Valdés King, González Cáceres J, Salisu Abdulkadir M. Prevalecia de depresión y factores de riesgo asociados a deterioro cognitivo en adultos mayores. Rev cuba med gen integr. 2018; 33(4).

Recibido: 9 de octubre de 2020

Aceptado: 23 de octubre de 2020 
